\title{
Suppressing H19 Modulates Tumorigenicity and Stemness in U251 and U87MG Glioma Cells
}

\author{
Weiguo $\mathrm{Li}^{1} \cdot$ Pengfei Jiang ${ }^{2} \cdot$ Xiaoling $\mathrm{Sun}^{2} \cdot$ Shujun $\mathrm{Xu}^{1} \cdot \mathrm{Xiangyu}_{\mathrm{Ma}^{1}} \cdot$ \\ Rucai Zhan ${ }^{3}$
}

Received: 7 October 2015/ Accepted: 18 December 2015/Published online: 16 March 2016

(C) The Author(s) 2016. This article is published with open access at Springerlink.com

\begin{abstract}
Glioblastoma multiforme (GBM) is a type of malignant carcinoma found in the brain. Its high frequency of occurrence and poor survival rate have garnered much research attention in recent years. Long non-coding RNAs (IncRNAs) are known to be related to the formation and progression of several cancer types by both promoting and suppressing tumor transformation. $\mathrm{H} 19$ is one such lncRNA and has been shown to be upregulated in a few types of cancer. In this study, we discovered that the expression of H19 increased in GBM cell lines. H19 knocked down GBM cells also displayed decreased cellular proliferation and a higher apoptosis rate when induced by temozolomide. Interestingly, the GBM cell lines U87MG and U251 were found to express cancer stem cell markers CD133, NANOG, Oct4 and Sox2. Expression of these markers was downregulated in H19-deficient cells. Collectively, these data suggest a role for H19 in contributing to GBM malignancy and the maintenance of its stem cell properties.
\end{abstract}

Keywords LncRNA · H19 · Glioblastoma ·

Glioma stem-like cells

Weiguo Li and Pengfei Jiang have contributed equally to this work.

Weiguo Li

leeweiguo_777@126.com

1 Neurosurgery Department, Qilu Hospital, Shandong University, 107 Wenhua West Road, Lixia District, Jinan 250012, Shandong, China

2 Yantai Yuhuangding Hospital, Yantai, China

3 Neurosurgery Department of No. 3 Hospital of Jinan, Jinan, China

\section{Introduction}

Glioblastoma multiforme (GBM), or glioblastoma, is a type of brain tumor arising from astrocytes, the starshaped glial cells that support and protect neural tissues in the brain and spinal cord (Furnari et al. 2007; Perry et al. 2004; Rosenblum 2007). Due to the extensive network of blood vessels in the brain, GBM cells proliferate rapidly, making GMB the most aggressive form of primary brain tumor (DeAngelis 2001; Maes and Van Gool 2011; Wrensch et al. 2002). GBM is the most prevalent brain tumor found in adults (DeAngelis 2001), and patients diagnosed with GBM usually have a poor prognosis; the median survival time is one year under standard treatment (Yan et al. 2013). Treatment typically involves surgery followed by radiotherapy. The addition of chemotherapy via temozolomide (TMZ) was shown to increase patients' survival period (Reardon et al. 2006; Stupp et al. 2005). However, the appearance of radio/chemotherapy-resistant GBM cells easily invalidates this treatment (Ahmed et al. 2013; Rich and Eyler 2008). Recent progress in cancer biology has identified that the resistant cells are mainly from a group expressing stem cell markers, which are defined as cancer stem cells (CSCs), or cancer-initiating cells (Clevers 2011; Lobo et al. 2007). CSCs are tumorigenic: a small population of CSCs is able to give rise to a tumor with heterogeneous cell types (Clevers 2011; Frank et al. 2010; Lobo et al. 2007; Price et al. 2008). In the particular case of GBM, these have been referred to as glioma stem cells (GSCs) (Frosina 2011; Ye et al. 2012). Although great effort has been made to study GSCs, their role in GBM remains largely unknown. Therefore, more research is necessary to identify the mechanisms that cause GBM and its reoccurrence in order for treatments to advance. 
One of the most promising targets in GBM treatment has been the discovery of the role of long non-coding RNA (lncRNA). Non-coding RNA is transcribed from non-coding DNA, which represents around $98 \%$ of human DNA and was initially considered "junk DNA" (International Human Genome Sequencing et al. 2001). LncRNA is defined as non-coding RNA of over 200 nucleotides in length (Gupta et al. 2010; Gutschner and Diederichs 2012; Zhu et al. 2011). Research has recently revealed the critical roles of lncRNAs in tumorigenesis and the progression of a few types of cancer, including GBM. Numerous lncRNAs have been identified at present (Kiang et al. 2015). Wang et al. reported that one lncRNA, colorectal neoplasia differentially expressed (CRNDE), was upregulated in glioma patients and cell lines. CRNDE promotes the proliferation and invasion of glioma cells by modulating the mTORsignaling pathway (Wang et al. 2015). Yao et al. showed that another lncRNA, X-inactive specific transcript (XIST), was also upregulated in glioma tissues and GSCs. The authors demonstrated the role of XIST in tumorigenesis by showing that the XIST-knockdown glioma cells displayed inhibited tumor growth, migration and invasion, and reduced apoptosis by regulating miR-152 (Yao et al. 2015).

Although a number of lncRNAs have been discovered that contribute to the formation and progression of GBM, the effort to look for differentially expressed lncRNAs in GBM continues. In the past 10 years, four independent groups have reported upregulation of H19 in GBM cells and GSCs. H19 was shown to encode an RNA transcript that was highly expressed in mouse embryos, and was later determined to be involved in cell proliferation in both mouse and human models (Bartolomei et al. 1991; Feil et al. 1994; Pachnis et al. 1984). Further studies demonstrated its involvement in GBM progression and invasion (Shi et al. 2013, 2014). However, the mechanisms involved remain unclear.

Our goal in this study was to examine the function of H19 in GBM as well as the underlying molecular mechanisms. To do this, we selected GBM cell lines and confirmed H19 upregulation in these cells. H19 was knocked down in several GBM cell lines, after which the cell proliferation rate, apoptosis, tumor formation capability, and expression of CSC markers were measured. We successfully identified several oncogenic functions of H19.

\section{Materials and Methods}

\section{Cell Culture}

Human glioblastoma cell lines U87MG, U251, U343, Hs683, LN215 and A172, and normal human astrocytes (NHA) cell line were cultured in Dulbecco's modified Eagle's medium (DMEM) (Invitrogen Life Technologies,
USA), supplemented with $10 \%$ fetal bovine serum (Gbico, USA), $100 \mathrm{U} / \mathrm{ml}$ penicillin, and $100 \mu \mathrm{g} / \mathrm{ml}$ streptomycin. All cells were incubated in a humidified atmosphere containing $5 \% \mathrm{CO}_{2}$ at $37{ }^{\circ} \mathrm{C}$.

\section{SiRNA Transfection}

Knockdown of H19 in all GBM cell lines was established via RNAi using a lentiviral vector. SiRNA oligonucleotides and the lentivirus were purchased from Ribo Biotech (Guangzhou, China). RT-PCR was performed after transduction to confirm the knockdown efficiency. SiRNA powders were dissolved in nuclease-free water to give a $50 \mu \mathrm{M}$ stock concentration and then diluted to a $10 \mathrm{nM}$ working concentration for all transfections. The ribonucleic acid sequences of siRNAs targeting H19 were as follows $\left(5^{\prime}-3^{\prime}\right)$ : sense, GCGGGUCUGUUUCUUUACUUU, antisense, AGUAAAGAAACAGACCCGCUU. The ribonucleic acid sequences of control siRNA were as follows $\left(5^{\prime}-\right.$ $3^{\prime}$ ): sense, GCGUUCUGGUCUUACUGUUUU, antisense, AGAGAAUAAACCCGCAGACUU. Transfection and cotransfection of siRNA were performed according to the manufacturer's protocol of lipofectamine 2000 (Invitrogen).

\section{RNA Extraction and RT-PCR}

The RNeasy Mini Kit (Qiagen) was used to extract the total RNA. RT-PCR was performed on an ABI PRISM 7500 (Applied Biosystems) with SYBR $^{\circledR}$ Premix Ex Taq ${ }^{\mathrm{TM}}$ (Perfect Real Time) (Takara). The oligonucleotide sequences used for RT-PCR analyses are shown in Table 1.

\section{Western Blot Analysis}

Cells were washed in ice cold phosphate-buffered saline (PBS) and then treated with a RIPA protein lysis buffer to prepare protein lysates as previously described (Pierce et al. 1999). The protein concentrations in the cell lysates were measured by Bio-Rad protein assay kits (Bio-Rad, Hercules, CA, USA) and then calibrated by standard bovine serum albumin concentrations. $40 \mu \mathrm{g}$ total proteins for each cell lysate sample were loaded and separated by SDS-PAGE on a $10 \%$ gel, and transferred to PVDF membranes (Bio-Rad). $5 \%$ bovine serum albumin in Tris buffer (TBS) was used for membrane blocking overnight at $4{ }^{\circ} \mathrm{C}$, and the membrane was then washed with PBS containing $0.1 \%$ Tween 20 (TBST). Primary antibodies specific to target proteins were used for probing for $1 \mathrm{~h}$ at room temperature and then washed with TBST again; corresponding secondary antibodies were used for detection. Finally, the membrane was subjected to autoradiography, and signals were quantified. Primary antibodies (mouse monoclonal) specific to CD133 and OCT4 were 
Table 1 Primers

\begin{tabular}{|c|c|c|}
\hline Gene & Primer & Product size (bp) \\
\hline Variant 1 & $\begin{array}{l}\text { Sense: GGCAAGAAGCGGGTCTGT } \\
\text { Anti-sense: GCTGCTGTTCCGATGGTGT }\end{array}$ & 273 \\
\hline Variant 2 & $\begin{array}{l}\text { Sense: GGCTCTGGAAGCTAGAGGAA } \\
\text { Anti-sense: CTGGGATGATGTGGTGGC }\end{array}$ & 168 \\
\hline Variant 3 & $\begin{array}{l}\text { Sense: GACCCAAGGACTCAAGCG } \\
\text { Anti-sense: GCGAGACTCCAGGAACACT }\end{array}$ & 115 \\
\hline GAPDH & $\begin{array}{l}\text { Sense: TGTGGGCATCAATGGATTTGG } \\
\text { Anti-sense: ACACCATGTATTCCGGGTCAAT }\end{array}$ & 116 \\
\hline CD133 & $\begin{array}{l}\text { Sense: AGTCGGAAACTGGCAGATAGC } \\
\text { Anti-sense: GGTAGTGTTGTACTGGGCCAAT }\end{array}$ & 99 \\
\hline Nanog & $\begin{array}{l}\text { Sense: TTTGTGGGCCTGAAGAAAACT } \\
\text { Anti-sense: AGGGCTGTCCTGAATAAGCAG }\end{array}$ & 116 \\
\hline Oct-04 & $\begin{array}{l}\text { Sense: GTGTTCAGCCAAAAGACCATCT } \\
\text { Anti-sense: GGCCTGCATGAGGGTTTCT }\end{array}$ & 156 \\
\hline Sox 2 & $\begin{array}{l}\text { Sense: TACAGCATGTCCTACTCGCAG } \\
\text { Anti-sense: GAGGAAGAGGTAACCACAGGG }\end{array}$ & 110 \\
\hline
\end{tabular}

purchased from MACS Miltenyi Biotec; NANOG antibody (rabbit polyclonal) was purchased from Santa Cruz Biotech; SOX2 antibody (goat polyclonal) was purchased from Santa Cruz Biotech; GAPDH antibody (mouse monoclonal) was purchased from Zhongshanjinqiao Biotech. Secondary antibodies were purchased from Zhongshanjinqiao Biotech. Film autoradiography reagents were purchased from Bio-Rad.

\section{CCK-8 Assay Analysis}

The CCK-8 solution was used to quantify the cell proliferation rate. Cells seeded in a 96-well plate were treated with $10 \mu \mathrm{l} \mathrm{CCK}-8$ solution, and incubated for $2 \mathrm{~h}$ at $37{ }^{\circ} \mathrm{C}$. The automated ELISA reader (Bio-Tek Instruments Inc., Winooski, VT, USA) was used to quantify absorbance $(A)$ of each well at $450 \mathrm{~nm}$, and the cell survival rate $(\%)$ was then calculated as follows:

$[A($ experiment $)-A($ blank $)] /[A($ control $)-A($ blank $)] * 100$.

\section{EdU Incorporation Assay}

EdU assay kit (Ribobio, Guangzhou China) was used to detect the proliferation status according to the manufacturers' instructions. Cells were briefly cultured in triplicate in confocal dishes at a density of $2 \times 10^{3}$ cells for $48 \mathrm{~h}$ at $37^{\circ} \mathrm{C}$, and $50 \mu \mathrm{M}$ of EdU was then added to each well. Cells were then cultured for an additional $2 \mathrm{~h}$ at $37^{\circ} \mathrm{C}$. The cells were then fixed with $4 \%$ formaldehyde for $15 \mathrm{~min}$ at room temperature and treated with $0.5 \%$ Triton $\mathrm{X}-100$ for $20 \mathrm{~min}$ at room temperature for permeabilization. After washing with PBS three times, $100 \mu \mathrm{l}$ of a $1 \times$ Apollo $^{\circledR}$ reaction cocktail was added to each well and the cells were incubated for $30 \mathrm{~min}$ at room temperature. The cells were then stained with $100 \mu$ l of Hoechst33342 for $30 \mathrm{~min}$ and visualized under a fluorescent microscope (Olympus Corporation, Tokyo, Japan).

\section{Flow Cytometry Analysis}

An Annexin V-PE apoptosis kit (BD Biosciences, Franklin Lakes, NJ, USA) was used to measure apoptosis. U87MG and U251 cells were first transfected with si-H19 or negative controls respectively, and the cells were then harvested and washed with ice cold PBS. The washed cells were re-suspended in an Annexin $\mathrm{V}$ binding buffer, followed by staining with Annexin V-PE and 7-AAD. The fluorescence signals of stained cells were then read by FACS (Becton-Dickinson, Franklin Lakes, NJ, USA), and the results were analyzed using Cell Quest software (Becton-Dickinson).

\section{Transwell Assay}

The invasion assay was performed using a Transwell chamber (Corning, NY, USA). $1 \times 10^{5}$ cells in serum-free medium were placed into the upper chamber with an insert coated with Matrigel (BD Biosciences, San Jose, CA, USA). Next, medium containing $10 \%$ FBS was added to the lower chamber. After $24 \mathrm{~h}$ of incubation, the cells remained on the upper membrane were eliminated, and the cells invaded through the membrane were fixed and stained with methanol and $0.1 \%$ crystal violet. The cells were imaged using an IX71 inverted microscope (Olympus, Tokyo, Japan). 


\section{Statistical Analysis}

All statistical analyses were performed with an unpaired Student's $t$ test. A $p<0.05$ was considered statistically significant.

\section{Results}

\section{Glioblastoma (GBM) Cells Showed Upregulated H19 Expression}

Since GBM cells are derived from normal astrocytes by malignant transformation, we first compared H19 expression in GBM cells and NHAs. To do this, H19 expression was measured in six different GBM cell lines: U87MG, U251, U343, Hs683, LN215 and A172. H19 expression in GBM cell lines was significantly higher than in the NHA cell line (Fig. 1a). Among the 6 GBM cell lines, U87MG cells showed a 44-fold upregulation of H19 expression compared to NHA cells, and U251 cells showed a 35-fold upregulation. Therefore, U87MG and U251 cell lines were chosen as representative GBM cell lines for the subsequent studies.

Next, we wanted to explore the function of H19 in GBM cells. To do this, H19 was knocked down by si-RNA in U87MG and U251 cell lines. The results indicated a $50 \%$ knockdown efficiency in U87MG and $70 \%$ efficiency in U251 (Fig. 1b).

\section{H19 Knockdown Reduced the Proliferation Rate and Migration Activity of GBM Cells}

By knocking down H19 in U87MG and U251 cell lines, we found that they both displayed decreased proliferation rates compared to non-treated cells and cells transfected with a non-specific control siRNA (Fig. 2a). Edu staining also confirmed a reduced proliferation rate in H19 knockdown GBM cells (Fig. 2b). These results indicate a role of H19 in the proliferation of GBM cells.

Next, we studied if H19 knockdown would also affect cell migration and invasion in GBM cell lines. We found that both U87MG and U251 cells displayed reduced cell mobility after H19 knockdown (Fig. 2c).

\section{H19 Knockdown Led to Increased TMZ-Induced Apoptosis in GBM Cells}

After determining that $\mathrm{H} 19$ reduced the proliferation rate of GBM cells, we wondered whether it would also increase apoptosis. To address this question, we induced apoptosis with temozolomide (TMZ), a widely used chemotherapeutic medicine to treat glioblastoma (He et al. 2015). The function of H19 in TMZ-induced GBM cell apoptosis was determined by comparing H19-knockdown GBM cells with normal GBM cells. The results showed that TMZ-treated U87MG and U251 cells with H19 knockdown had a larger proportion undergoing apoptosis compared to non-treated cells or TMZ-treated cells transfected with non-specific control siRNA (Fig. 3a). Most of the apoptotic cells were found in the early stages, as indicated by Annexin V positive and PI negative staining (Fig. 3a). Cells with H19 knockdown had a slightly higher number in necrosis, indicated by double positive staining, compared to the negative controls (Fig. 3a). This result suggested the involvement of $\mathrm{H} 19$ in the anti-apoptosis process of GBM cells.

Since H19-knocked down GBM cells showed signs of apoptosis in staining, we sought to confirm this by measuring the level of metabolic markers in apoptosis. Our results showed that TMZ-induced U87MG and U251 cells with H19 knockdown had reduced pro-caspase 3 and elevated cleaved caspase 3 expression compared to the negative controls. Similarly, H19 knockdown caused increased

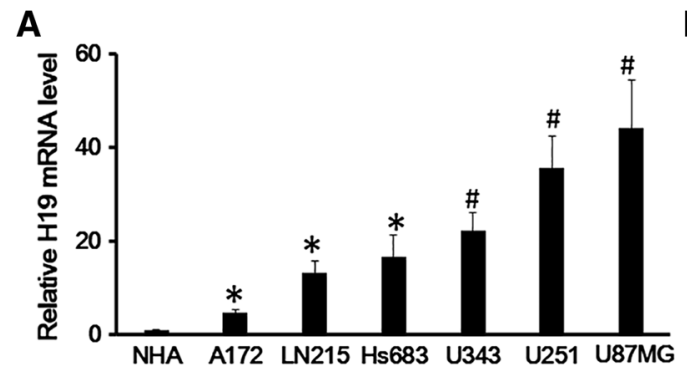

Fig. 1 Glioblastoma (GBM) cell lines showed upregulated H19 expression. a The lysates of GBM cells from A172, LN215, Hs683, U343, U251 and U87MG cell lines were harvested and examined for H19 expression; the cell lysates of Normal Human Astrocytes (NHA) cell line were used as the negative control. b The efficiency of H19
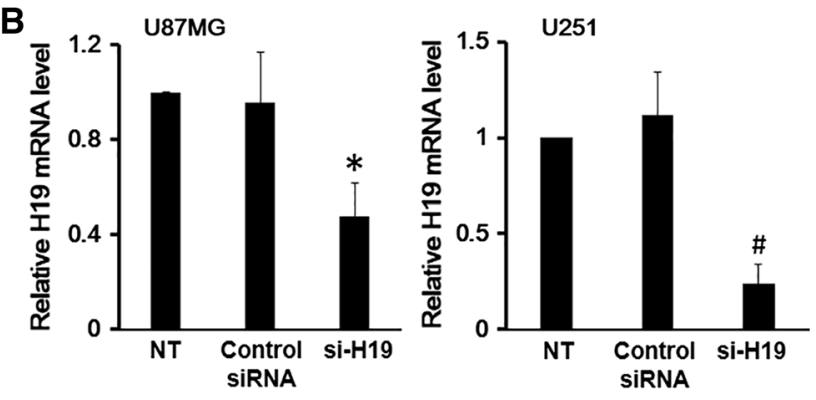

knockdown by siRNA in U87MG and U251 cells was measured by qRT-PCR. Non-treated (NT) U87MG and U251 cells and cells transfected with control siRNA were included as controls. Data here represent an average of three independent experiments; the error bar indicates the standard deviation of the mean; * $p<0.05$; ${ }^{*} p<0.005$ 
A

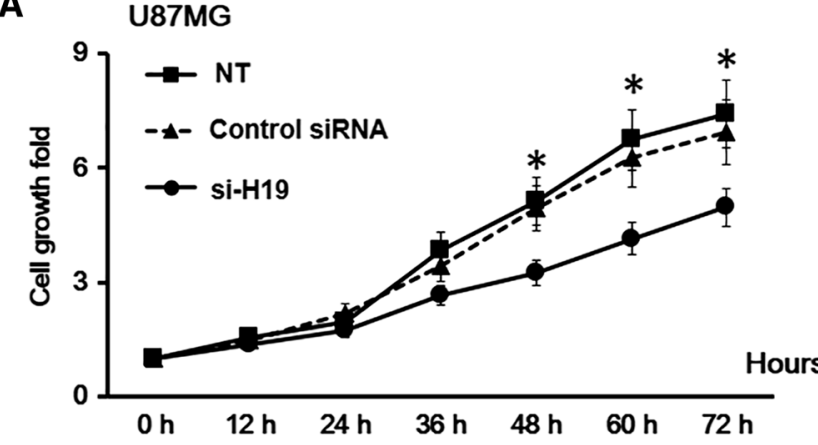

B

U87MG

NT

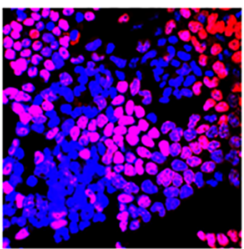

C

U87MG

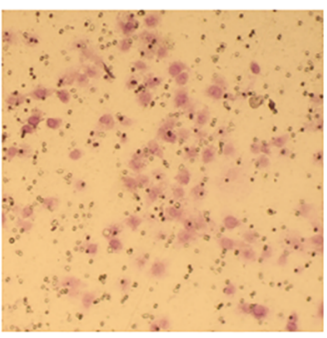

Control siRNA
Control siRNA
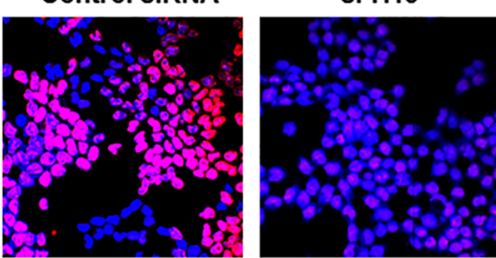

U251
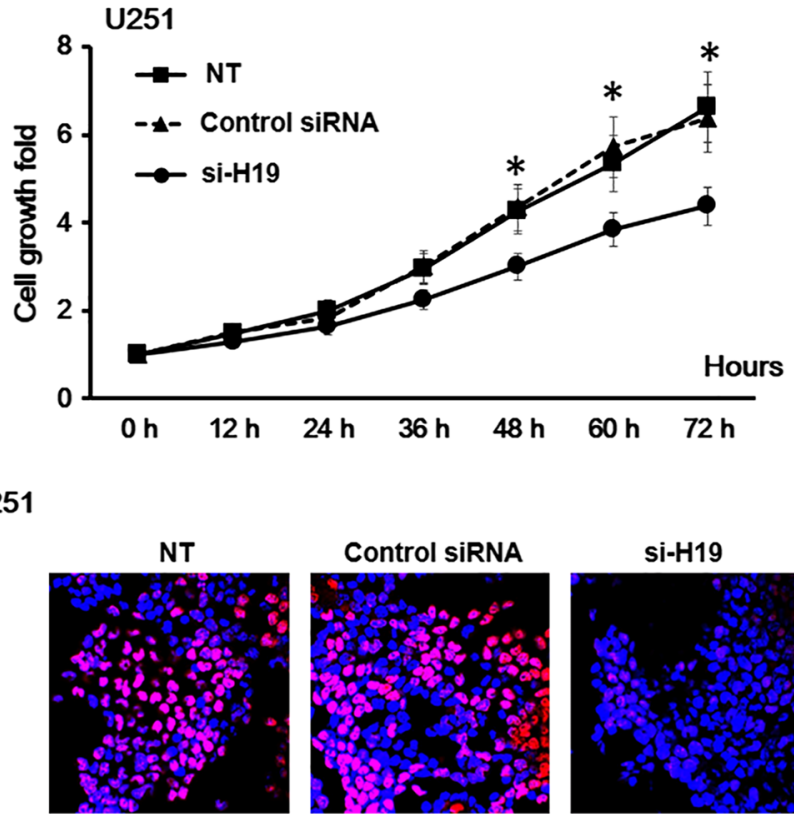

Control siRNA

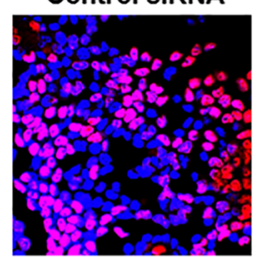

si-H19

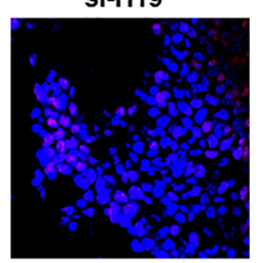

U251

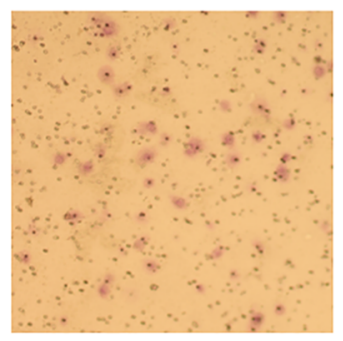

si-H19

Fig. 2 H19 knockdown decreased the proliferation rate of glioblastoma (GBM) cells. a The proliferation of U87MG and U251 cells with $\mathrm{H} 19$ knockdown was measured by CCK-8 assay. U87MG and U251 cells transfected with non-specific control siRNA and no siRNA (NT) were both included as negative controls. Data here represent an average of three independent experiments; error bar indicates standard deviation of the mean; $* p<0.05 ;{ }^{*} p<0.005$. b The proliferation of U87MG and U251 cells with H19 knockdown was measured by EdU assay. U87MG and U251 cells transfected with

expression of Bax, and decreased the Bcl-2 level in TMZinduced U87MG and U251 cells (Fig. 3b). These data confirmed that H19 knockdown led to increased apoptosis of GBM cells under TMZ treatment.

\section{Cancer Stem Cell Markers were Downregulated in GBM Cells with H19 Knockdown}

We have so far shown that H19 knockdown impaired the proliferation of GBM cells and also increased their apoptosis under TMC induction. By examining H19 knockdown cells, we found that they adopted a rounded shape and became less attached, which led us to wonder whether they harbor stem cell properties. To investigate this, both the

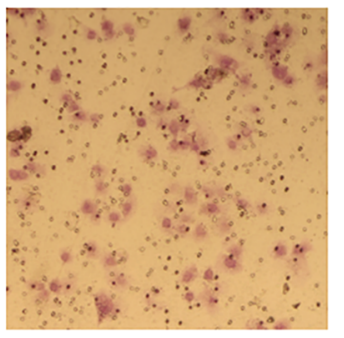

Control siRNA

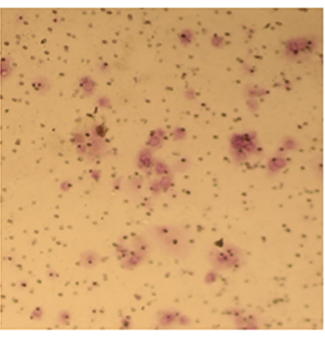

si-H19 non-specific control siRNA and no siRNA (NT) were both included as negative controls. Three independent experiments were done; representative fluorescent microscope images were shown here. c The invasion of U87MG and U251 cells with H19 knockdown was measured by Transwell assay. U87MG and U251 cells transfected with non-specific control siRNA and no siRNA (NT) were both included as negative controls. Five pictures were collected for each group, and the representative images were shown here

mRNA and protein levels of four stem cell markers were measured in U87MG and U251 cells, and they were all found to be positive (Fig. 4a, b). However, H19 knockdown in these cells reduced the expression of the four markers in both mRNA (Fig. 4a) and protein levels (Fig. 4b). These findings suggest that the oncogenic properties of H19 may be due to transforming differentiated astrocytes into GSCs.

Next, we examined whether TMZ would also affect stem cell marker expression in GMB cell lines. To do this, we divided both U87MG and U251 cells into three groups. The first group was treated with TMZ alone; the second was transfected with control siRNA and treated with TMZ; and the third was transfected with H19 siRNA and treated 
A
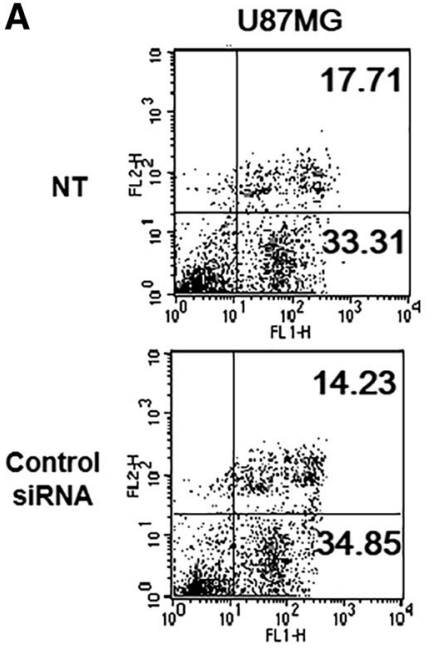

si-H19

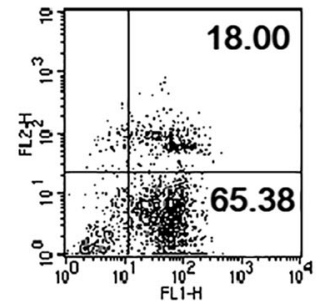

$\mathrm{U} 251$
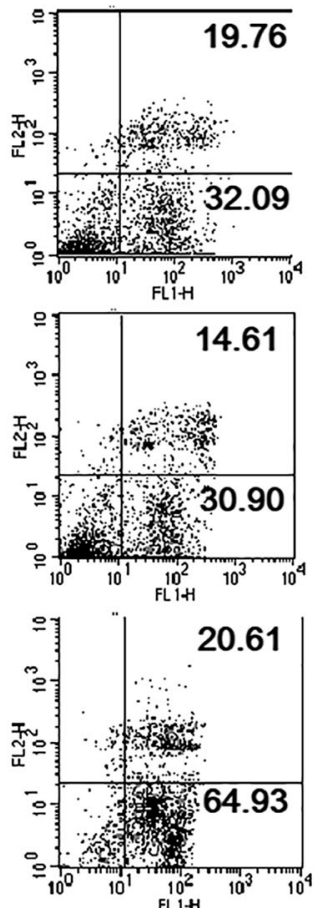

Fig. 3 H19 knockdown led to apoptosis in glioblastoma (GBM) cells. a Apoptosis of U87MG and U251 cells with H19 knockdown was measured by Annexin V-FITC/PI double staining assay. TMZtreated U87MG and U251 cells transfected with non-specific control siRNA and non-treated (NT) were both included as negative controls. Three independent experiments were done; representative flow

with TMZ. We found that TMZ treatment alone and TMZtreated cells transfected with control siRNA showed a very similar expression level of the four stem cell markers, while TMZ-treated cells transfected with H19 siRNA showed significantly reduced expression, which was about $40-60 \%$ of the expression in the other two conditions (Fig. 4c).

\section{Discussion}

Using representative cell lines, we examined the role of H19 in GBM. We found that H19 promoted cell proliferation in GBM since U87MG and U251 GBM cells with H19 knockdown exhibited a reduced cell proliferation rate (Fig. 2). In addition, we showed that TMZ-induced apoptosis increased in U87MG and U251 GBM cells with H19 knockdown (Fig. 3), which suggested the anti-apoptosis function of H19 in GBM. Finally, a screening of stem cell markers found that their expression dropped significantly in H19-deficient GBM cells (Fig. 4), indicating the involvement of $\mathrm{H} 19$ in the maintenance of the GSC population.

B

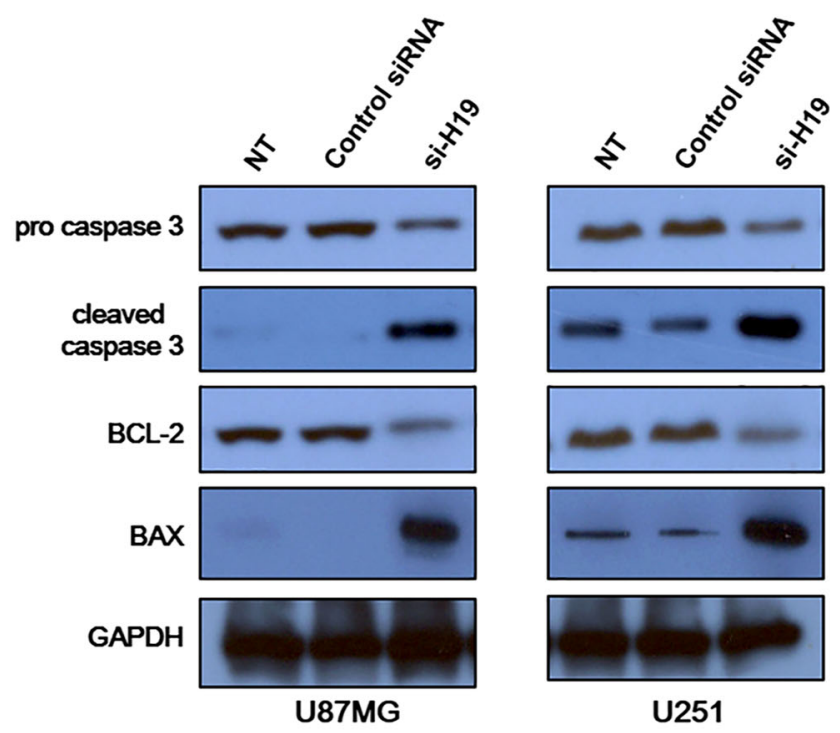

cytometry data were shown here. b Protein expression level of pro caspase 3, cleaved caspase 3, BCL-2 and BAX was measured in U87MG and U251 cells with H19 knockdown. U87MG and U251 cells transfected with non-specific control siRNA and no siRNA (NT) were both included as negative controls

Our study successfully established a correlation between H19 and the proliferation of GBM cells. H19 has been known for its involvement in cell proliferation in mouse embryos since shortly after its discovery three decades ago (Pachnis et al. 1984). Later research identified H19 in human cells and found a close link with insulin-like growth factor 2 (Igf2) via their reciprocal imprinting in embryos (Feil et al. 1994; Zhang and Tycko 1992). However, these imprinting studies revealed that H19 functioned to downregulate cellular proliferation (Bartolomei et al. 1991; Feil et al. 1994), which was contradictory to our discovery in GBM cell lines. Similarly, the function of H19 in cancer is also in debate. Previous studies have shown that $\mathrm{H} 19$ bears both oncogenic (Adriaenssens et al. 1998; Moulton et al. 1994) and tumor-suppressive properties (Hao et al. 1993; Yoshimizu et al. 2008). However, recent research has supported the former role by demonstrating upregulation of H19 in a few types of cancer and its involvement in promoting cancer invasion, migration and metastasis (Huang et al. 2015; Liu et al. 2015; Yang et al. 2015; Zhou et al. 2015). Here we found that H19 was upregulated in GBM cells, especially those in a late-stage, since the U87MG cell line originated from a stage-IV GBM patient. These 
A

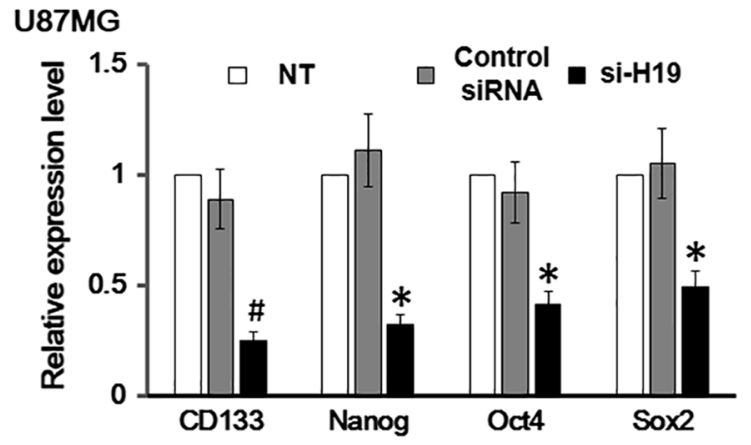

U251

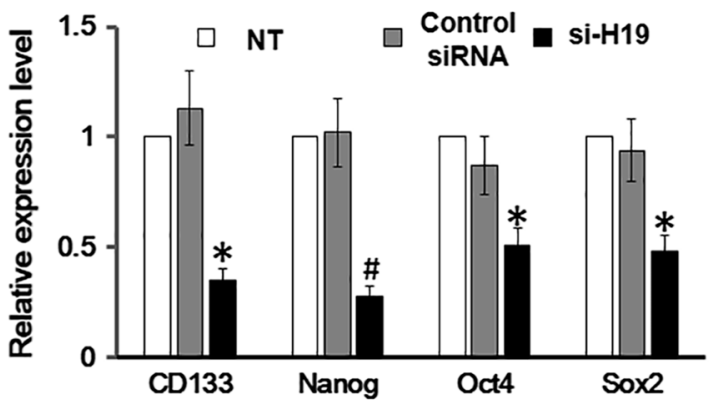

U87MG

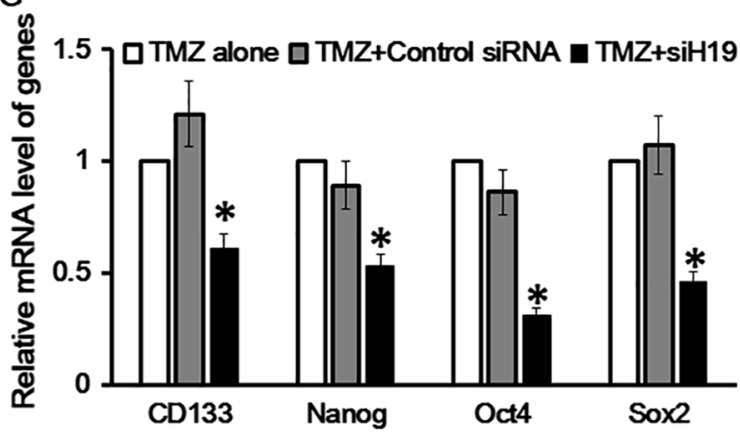

Fig. 4 Cancer stem cell (GSC) markers were downregulated in glioblastoma (GBM) cells with H19 knockdown. a mRNA level of CD133, Nanog, Oct-4 and Sox2 was measured in U87MG and U251 cells with H19 knockdown. U87MG and U251 cells transfected with non-specific control siRNA and no siRNA (NT) were both included as negative controls. Data here represent an average of three independent experiments; error bar indicates standard deviation of the mean; ${ }^{*} p<0.05 ;{ }^{*} p<0.005$. b Protein level of CD133, Nanog, Oct-4 and Sox 2 was measured in U87MG and U251 cells with H19 knockdown.

findings support the oncogenic function of H19 in tumor formation and development. However, it is worth noting that these seemingly contradictory roles of $\mathrm{H} 19$ were found in different types of cancer. It is likely that H19 plays different roles in different tissues or developmental stages, and its role in a specific tissue remains the same in both normal and tumor cells. For example, H19 was shown to repress cellular growth in embryos and was also found to be a tumor suppresser in embryonic carcinoma (Hao et al. 1993).

Besides mediating cell proliferation, $\mathrm{H} 19$ was also found to be responsible for anti-apoptosis in GBM cells in this
B

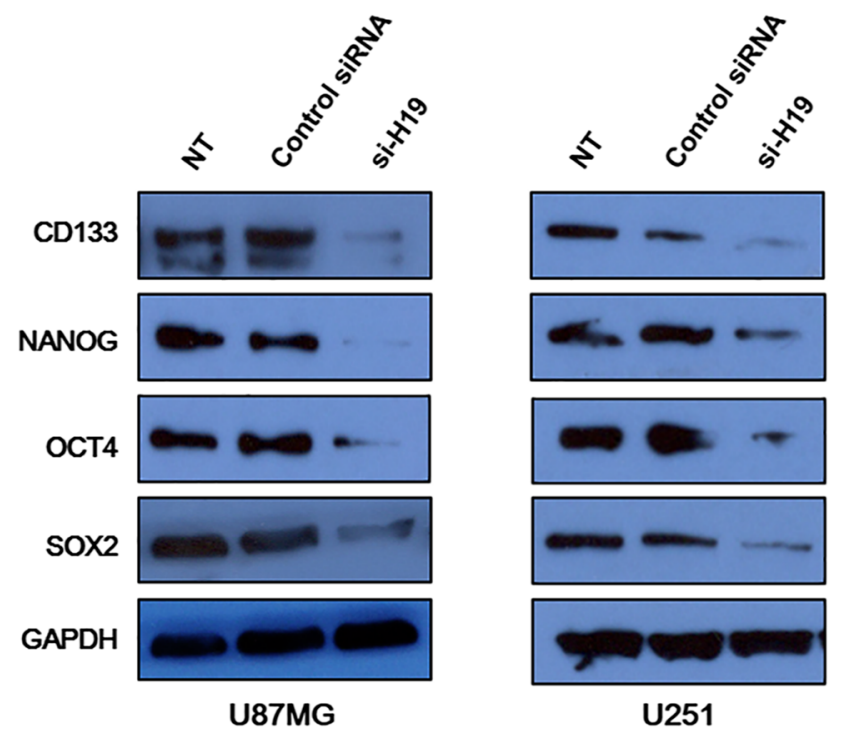

U251

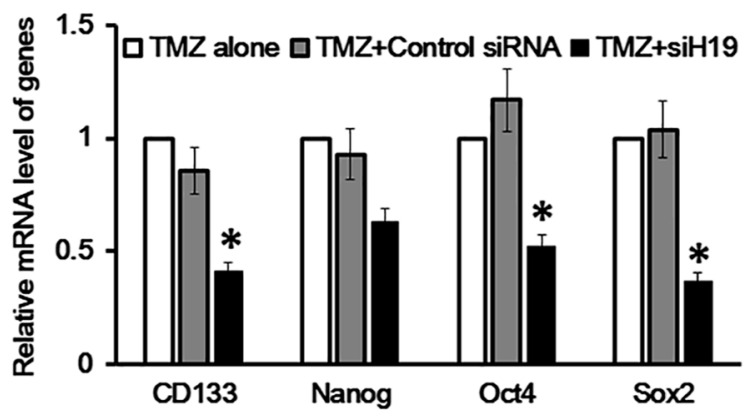

U87MG and U251 cells transfected with non-specific control siRNA and no siRNA (NT) were both included as negative controls. $\mathbf{c}$ mRNA level of CD133, Nanog, Oct-4 and Sox2 was measured in U87MG and U251 cells treated with temozolomide (TMZ). TMZ-alone and $\mathrm{TMZ}+$ control siRNA groups were both included as negative controls. Data here represent an average of three independent experiments; error bar indicates standard deviation of the mean; $* p<0.05$

study. We found that twice the number of GBM cells with H19 knockdown experienced apoptosis compared to normal GBM cells under TMZ treatment (Fig. 3a). No difference was found in the number of cells undergoing late apoptosis, and only slightly more H19-deficient cells were found in necrosis in H19-knocked down cells (Fig. 3a). However, with longer treatment or a higher dose of TMZ, a greater percentage of cells are likely to be identified in these two stages. By studying metabolic markers in apoptosis, we found increased Bax expression and decreased Bcl-2 expression in GBM cells with H19 knockdown. Bax 
is a pro-apoptosis protein and is activated by the tumor suppressor p53 (Miyashita and Reed 1995).

In the final part of this study, we found that GBM cell lines U87MG and U251 both had CSC markers CD133, Nanog, Oct4 and Sox2. H19-knocked down cell lines showed significantly reduced expression of these markers. This finding confirms the theory that GBM cells originate from CSCs (Piccirillo et al. 2009), and also suggests that over-expression of $\mathrm{H} 19$ might function to transform normal astrocytes into GSCs. However, this possibility still needs further investigation. Previous research identified that overexpression of H19 in CD133+ GBM cells increased their neurosphere formation and tumor growth (Jiang et al. 2015), which showed the importance of H19 in GSCs. Our results further demonstrate the cancer-promoting role of H19 in GSCs and help expand the existing knowledge from CD133+ GBM cells to ones bearing four different stem cell markers. However, our results do not distinguish the GSC populations bearing these markers. In other words, there may be four GSC populations, each expressing one of the four stem cell markers; but there may also be populations expressing a combination of these four stem cell markers. Further studies on H19 expression and its function on these distinguished GSC populations should prove beneficial. Moreover, the relationship between H19 and the four stem cells markers merits further investigation as they are involved in several important cellular signaling pathways such as PI3K/Akt, p53/Rb and Wnt/ß-catenin, malfunction of which usually leads to cancer cell transformation.

The involvement of H19 in maintenance of stem cell markers has been reported in other types of cancer before (Ben-Porath et al. 2008; Gabory et al. 2009; Jiang et al. 2015). Although no detailed mechanisms were shown, we hypothesize that $\mathrm{H} 19$ promotes the expression of stem cell markers via regulating the Lin28/Let-7 pathway, which has been shown to maintain stem cell properties in a number of studies. By studying H19 in muscle tissue, where the H19 level is maintained relatively high, a group of researchers found that H19 inhibited Let-7 expression via direct binding, and depletion of $\mathrm{H} 19$ accelerated muscle cell differentiation, which suggested a role of H19 in maintaining stem cell properties (Gao et al. 2014). A recent publication reported that suppressing Let- 7 increased Oct4 and Sox 2 expression in CSC-like oral squamous cell carcinoma (OSCC) by targeting ARID3B and HMGA2, which directly interact with Oct4 and Sox2. The same publication also showed that Lin28 affected the methylation status on Oct4 and Nanog promoter in reprogramming HNOK cells to HNOK-iPSCs (Chien et al. 2015). Although no direct evidence has been found to show the correlation between Let-7 and CD133, several groups reported downregulated Let-7 level in CD133+ positive CSC like cells (Bao et al. 2014; Luo et al. 2013).
Finally, not only did this study reveal the function of H19 in GBM cells, it also added evidence supporting the role of lncRNA in cancer. Besides H19, other lncRNAs have been found to either positively or negatively regulate tumorigenesis and metastasis. Therefore, further studies on lncRNAs and its link to cancer are called for, which could lead to new treatments and perhaps 1 day a cure for cancer.

\section{Compliance with Ethical Standards}

Conflict of interest All the authors declare no conflict of interest.

Open Access This article is distributed under the terms of the Creative Commons Attribution 4.0 International License (http://crea tivecommons.org/licenses/by/4.0/), which permits unrestricted use, distribution, and reproduction in any medium, provided you give appropriate credit to the original author(s) and the source, provide a link to the Creative Commons license, and indicate if changes were made.

\section{References}

Adriaenssens E et al (1998) H19 overexpression in breast adenocarcinoma stromal cells is associated with tumor values and steroid receptor status but independent of $\mathrm{p} 53$ and $\mathrm{Ki}-67$ expression. Am J Pathol 153:1597-1607. doi:10.1016/S0002-9440(10)65748-3

Ahmed AU, Auffinger B, Lesniak MS (2013) Understanding glioma stem cells: rationale, clinical relevance and therapeutic strategies. Expert Rev Neurother 13:545-555. doi:10.1586/ern.13.42

Bao B et al (2014) Differentially expressed miRNAs in cancer-stemlike cells: markers for tumor cell aggressiveness of pancreatic cancer. Stem Cells Dev 23:1947-1958. doi:10.1089/scd.2013. 0551

Bartolomei MS, Zemel S, Tilghman SM (1991) Parental imprinting of the mouse H19 gene. Nature 351:153-155. doi:10.1038/351153a0

Ben-Porath I, Thomson MW, Carey VJ, Ge R, Bell GW, Regev A, Weinberg RA (2008) An embryonic stem cell-like gene expression signature in poorly differentiated aggressive human tumors. Nat Genet 40:499-507. doi:10.1038/ng.127

Chien CS et al (2015) Lin28B/Let-7 regulates expression of Oct4 and Sox 2 and reprograms oral squamous cell carcinoma cells to a stem-like state. Cancer Res 75:2553-2565. doi:10.1158/00085472.CAN-14-2215

Clevers H (2011) The cancer stem cell: premises, promises and challenges. Nat Med 17:313-319. doi:10.1038/nm.2304

DeAngelis LM (2001) Brain tumors. N Engl J Med 344:114-123. doi:10.1056/NEJM200101113440207

Feil R, Walter J, Allen ND, Reik W (1994) Developmental control of allelic methylation in the imprinted mouse Igf2 and H19 genes. Development 120:2933-2943

Frank NY, Schatton T, Frank MH (2010) The therapeutic promise of the cancer stem cell concept. J Clin Invest 120:41-50. doi:10. 1172/JCI41004

Frosina G (2011) Frontiers in targeting glioma stem cells. Eur J Cancer 47:496-507. doi:10.1016/j.ejca.2010.11.017

Furnari FB et al (2007) Malignant astrocytic glioma: genetics, biology, and paths to treatment. Genes Dev 21:2683-2710. doi:10.1101/gad.1596707

Gabory A et al (2009) H19 acts as a trans regulator of the imprinted gene network controlling growth in mice. Development 136:3413-3421. doi:10.1242/dev.036061 
Gao Y et al (2014) The H19/let-7 double-negative feedback loop contributes to glucose metabolism in muscle cells. Nucleic Acids Res 42:13799-13811. doi:10.1093/nar/gku1160

Gupta RA et al (2010) Long non-coding RNA HOTAIR reprograms chromatin state to promote cancer metastasis. Nature 464:1071-1076. doi:10.1038/nature08975

Gutschner T, Diederichs S (2012) The hallmarks of cancer: a long non-coding RNA point of view. RNA Biol 9:703-719. doi:10. 4161/rna.20481

Hao Y, Crenshaw T, Moulton T, Newcomb E, Tycko B (1993) Tumour-suppressor activity of H19 RNA. Nature 365:764-767. doi:10.1038/365764a0

$\mathrm{He} \mathrm{H}$ et al (2015) MEK2 is a prognostic marker and potential chemosensitizing target for glioma patients undergoing temozolomide treatment. Cell Mol Immunol. doi:10.1038/cmi.2015.46

Huang C et al (2015) Upregulation of H19 promotes invasion and induces epithelial-to-mesenchymal transition in esophageal cancer. Oncol Lett 10:291-296. doi:10.3892/ol.2015.3165

International Human Genome Sequencing $C$ et al (2001) Initial sequencing and analysis of the human genome. Nature 409:860-921. doi:10.1038/35057062

Jiang X et al (2015) Increased level of H19 long noncoding RNA promotes invasion, angiogenesis, and stemness of glioblastoma cells. J Neurosurg 124:129-136. doi:10.3171/2014.12.JNS1426

Kiang KM, Zhang XQ, Leung GK (2015) Long non-coding RNAs: the key players in glioma pathogenesis. Cancers (Basel) 7:1406-1424. doi:10.3390/cancers7030843

Liu C, Chen Z, Fang J, Xu A, Zhang W, Wang Z (2015) H19-derived miR-675 contributes to bladder cancer cell proliferation by regulating p53 activation. Tumour Biol. doi:10.1007/s13277015-3779-2

Lobo NA, Shimono Y, Qian D, Clarke MF (2007) The biology of cancer stem cells. Annu Rev Cell Dev Biol 23:675-699. doi:10. 1146/annurev.cellbio.22.010305.104154

Luo G et al (2013) Highly lymphatic metastatic pancreatic cancer cells possess stem cell-like properties. Int J Oncol 42:979-984. doi:10.3892/ijo.2013.1780

Maes W, Van Gool SW (2011) Experimental immunotherapy for malignant glioma: lessons from two decades of research in the GL261 model. Cancer Immunol Immunother 60:153-160. doi:10.1007/s00262-010-0946-6

Miyashita T, Reed JC (1995) Tumor suppressor p53 is a direct transcriptional activator of the human bax gene. Cell 80:293-299

Moulton T et al (1994) Epigenetic lesions at the H19 locus in Wilms' tumour patients. Nat Genet 7:440-447. doi:10.1038/ng0794-440

Pachnis V, Belayew A, Tilghman SM (1984) Locus unlinked to alpha-fetoprotein under the control of the murine raf and Rif genes. Proc Natl Acad Sci USA 81:5523-5527

Perry A, Aldape KD, George DH, Burger PC (2004) Small cell astrocytoma: an aggressive variant that is clinicopathologically and genetically distinct from anaplastic oligodendroglioma. Cancer 101:2318-2326. doi:10.1002/cncr.20625

Piccirillo SG, Binda E, Fiocco R, Vescovi AL, Shah K (2009) Brain cancer stem cells. J Mol Med (Berl) 87:1087-1095. doi:10.1007/ s00109-009-0535-3

Pierce AJ, Johnson RD, Thompson LH, Jasin M (1999) XRCC3 promotes homology-directed repair of DNA damage in mammalian cells. Genes Dev 13:2633-2638
Price ND, Foltz G, Madan A, Hood L, Tian Q (2008) Systems biology and cancer stem cells. J Cell Mol Med 12:97-110. doi:10.1111/j. 1582-4934.2007.00151.x

Reardon DA, Rich JN, Friedman HS, Bigner DD (2006) Recent advances in the treatment of malignant astrocytoma. J Clin Oncol 24:1253-1265. doi:10.1200/JCO.2005.04.5302

Rich JN, Eyler CE (2008) Cancer stem cells in brain tumor biology. Cold Spring Harb Symp Quant Biol 73:411-420. doi:10.1101/ sqb. 2008.73 .060

Rosenblum MK (2007) The 2007 WHO classification of nervous system tumors: newly recognized members of the mixed glioneuronal group. Brain Pathol 17:308-313. doi:10.1111/j. 1750-3639.2007.00079.x

Shi X, Sun M, Liu H, Yao Y, Song Y (2013) Long non-coding RNAs: a new frontier in the study of human diseases. Cancer Lett 339:159-166. doi:10.1016/j.canlet.2013.06.013

Shi Y et al (2014) Long non-coding RNA H19 promotes glioma cell invasion by deriving miR-675. PLoS One 9:e86295. doi:10. 1371/journal.pone.0086295

Stupp R et al (2005) Radiotherapy plus concomitant and adjuvant temozolomide for glioblastoma. N Engl J Med 352:987-996. doi:10.1056/NEJMoa043330

Wang Y, Wang Y, Li J, Zhang Y, Yin H, Han B (2015) CRNDE, a long-noncoding RNA, promotes glioma cell growth and invasion through mTOR signaling. Cancer Lett 367:122-128. doi:10. 1016/j.canlet.2015.03.027

Wrensch M, Minn Y, Chew T, Bondy M, Berger MS (2002) Epidemiology of primary brain tumors: current concepts and review of the literature. Neuro Oncol 4:278-299

Yan Y et al (2013) SAMSN1 is highly expressed and associated with a poor survival in glioblastoma multiforme. PLoS One 8:e81905. doi:10.1371/journal.pone.0081905

Yang Z, Lu Y, Xu Q, Tang B, Park CK, Chen X (2015) HULC and H19 played different roles in overall and disease-free survival from hepatocellular carcinoma after curative hepatectomy: a preliminary analysis from gene expression omnibus. Dis Markers 2015:191029. doi:10.1155/2015/191029

Yao Y et al (2015) Knockdown of long non-coding RNA XIST exerts tumor-suppressive functions in human glioblastoma stem cells by up-regulating miR-152. Cancer Lett 359:75-86. doi:10.1016/ j.canlet.2014.12.051

Ye XZ et al (2012) Tumor-associated microglia/macrophages enhance the invasion of glioma stem-like cells via TGF-beta1 signaling pathway. J Immunol 189:444-453. doi:10.4049/ jimmunol.1103248

Yoshimizu T et al (2008) The H19 locus acts in vivo as a tumor suppressor. Proc Natl Acad Sci USA 105:12417-12422. doi:10. 1073/pnas.0801540105

Zhang Y, Tycko B (1992) Monoallelic expression of the human H19 gene. Nat Genet 1:40-44. doi:10.1038/ng0492-40

Zhou X, Ye F, Yin C, Zhuang Y, Yue G, Zhang G (2015) The Interaction Between MiR-141 and IncRNA-H19 in Regulating Cell Proliferation and Migration in Gastric Cancer. Cell Physiol Biochem 36:1440-1452. doi:10.1159/000430309

Zhu Y et al (2011) ncRAN, a newly identified long noncoding RNA, enhances human bladder tumor growth, invasion, and survival. Urology 77:510.e1-510.e5. doi:10.1016/j.urology.2010.09.022 